U. of Iowa $90-19$

\title{
Whistlers in Neptune's Magnetosphere: \\ Evidence of Atmospheric Lightning \\ by
}

D. A. Gurnett, W. S. Kurth,

I. H. Cairns, and L. J. Granroth

June 1990

Department of Physics and Astronomy

The University of Iowa

Iowa City, IA 52242 


$$
\text { — } 2 \text { - }
$$


ABSTRACT

During the Voyager 2 flyby of Neptune, a series of 16 whistlerlike events were detected by the plasma wave instrument near closest approach. These events were observed at radial distances from 1.30 to $1.99 \mathrm{R}_{\mathrm{N}}$ and magnetic latitudes from $-7^{\circ}$ to $33^{\circ}$. The frequencies ranged from 6.1 to $12.0 \mathrm{kHz}$, and the dispersions fit the Eckersley law for lightning-generated whistlers. Lightning in the atmosphere of Neptune is the only known source of such signals. The frequency range of the whistlers (up to $12 \mathrm{kHz}$ ) indicates that the local electron densities are substantially higher $\left(\mathrm{N}_{e}>30\right.$ to $\left.100 \mathrm{~cm}^{-3}\right)$ than indicated by the in situ plasma measurements. The dispersion of the whistlers is very large, typically 26,000 sec $\mathrm{Hz}^{1 / 2}$. Based on existing plasma density models and measurements, the dispersions are too large to be accounted for by a single direct path from the lightning source to the spacecraft. Therefore, multiple bounces from one hemisphere to the other are required. The most likely propagation path probably involves a lightning source on the dayside of the planet, with repeated bounces through the dense dayside ionosphere at low L-values. 



\section{INTRODUCTION}

The Voyager 2 spacecraft, which flew by Neptune on August 25, 1989, included a plasma wave instrument that provided the first measurements of low frequency $(10 \mathrm{~Hz}$ to $56 \mathrm{kHz}$ ) plasma waves in the magnetosphere of Neptune [Gurnett et al., 1989]. During the flyby of Neptune, the plasma wave instrument detected a series of highly dispersed signals that are believed to be whistlers produced by lightning in the atmosphere of Neptune. The purpose of this paper is to describe the observations and to discuss the initial interpretation of these signals.

Whistlers, as is well known, are electromagnetic waves excited by lightning that propagate through the magnetosphere at frequencies below the electron cyclotron frequency and electron plasma frequency. The mode of propagation of these signals is known as the whistler mode [Stix, 1962]. Since the index of refraction of the magnetospheric plasma depends on frequency, the impulsive signal from a lightning flash is converted into a whistling tone as the wave propagates through the plasma, hence the term "whistler." The wave energy tends to be guided along the magnetic field and in some cases can bounce back and forth along the magnetic field lines from one hemisphere to the other. Terrestrial whistlers were first reported by Barkhausen [1919]. The dispersive properties of these signals were later studied in considerable detail by Eckersley [1935], and the first comprehensive theory of 
whistler propagation was developed by Storey [1953]. For a review of the observations and theory of terrestrial whistlers, see Helliwell [1965].

Prior to the Neptune encounter three of the outer planets, Jupiter, Saturn and Uranus, were known to have atmospheric lightning. The discovery of lightning at these planets came from a combination of imaging [Smith et al., 1979], radio measurements [Kaiser et al., 1983; Zarka and Pedersen, 1986], and whistler observations [Scarf et al., 1979; Gurnett et al., 1979; Menietti and Gurnett, 1980; and Kurth et al., 1985]. Since lightning appears to be a common feature of the outer planets, it is not surprising that lightning has now been discovered at Neptune. In fact, Borucki [1989] has made specific predictions regarding the occurrence of lightning in the atmosphere of Neptune. According to his calculations, the lightning flash rate at Neptune is estimated to be $2.0 \times 10^{-4}$ flashes $\mathrm{km}^{-2} \mathrm{yr}^{-1}$, which is a factor of 19 smaller than at Jupiter. 


\section{OBSERVATIONS}

During the Voyager 2 flyby of Neptune, a total of 16 whistler-like events were detected by the plasma wave instrument. These events were all identified using data from the wideband waveform receiver. The wideband receiver provides measurements of the electric field waveform over a frequency range from $50 \mathrm{~Hz}$ to $12 \mathrm{kHz}$ at a sample rate of 28,800 samples per second. For a description of the plasma wave instrument, see Scarf and Gurnett [1977]. Since the data rate generated by the wideband receiver is very high, $115.2 \mathrm{kbits} / \mathrm{s}$, these measurements can only be obtained during a limited number of periods called wideband frames. A wideband frame normally consists of 48 seconds of data. However, because of time sharing with the imaging system, in some cases the duration of the wideband signal at Neptune was as short as 5 seconds.

The wideband frames obtained in the region around closest approach, which is where the whistler-like signals were observed, are summarized in Figure 1. This illustration shows the spacecraft trajectory as a function of radial distance, $R$, and magnetic dipole latitude, $\lambda_{m}$. The radial distance is given in Neptune radii, where $1 R_{N}=24,762 \mathrm{~km}$. The magnetic latitude was computed using the of fset tilted dipole (OTD) magnetic field model described by Ness et al. [1989], using the most recent parameters (known as OTD 2) [N. F. Ness, personal communication, 1990]. The circles along the trajectory show 
the locations of all the wideband frames obtained near closest approach. All of these are 48-second frames, except for the 5 frames on the inbound leg near $60-70^{\circ}$ magnetic latitude, which are 5-second frames. The open circles indicate frames in which no whistlers were observed, and the solid black circles indicate frames in which whistlers were observed. Each whistler has been given an identification number, from 1 to 16. These numbers are listed in Figure 1 next to the wideband frame in which the whistler was observed. As can be seen, all of the whistlers were observed near the planet, $R$ $<2 \mathrm{R}_{\mathrm{N}}$, and at low magnetic latitudes, $-7^{\circ}<\lambda_{\mathrm{m}}<33^{\circ}$. The whistler occurrence rate, computed by dividing the total number of whistlers by the total amount of wideband time, is approximately 1.6 whistlers per minute.

The whistler-like events listed in Figure 1 were all identified by visually inspecting frequency-time spectrograms of the wideband data. These spectrograms were generated by Fourier-transforming successive 60-msec segments of the electric field waveform, and displaying the Fourier amplitudes in the form of a spectrogram. Any feature, no matter how weak, that consisted of a narrowband tone decreasing in frequency with increasing time, was identified as a whistler. Roughly half of the events are very weak and require special viewing equipment (back-lighted slides) to be seen. The remaining events are clear and easily identifiable on normal black and white or color prints.

Two spectrograms with easily identifiable whistlers are shown in Figures 2 and 3 . Figure 2 shows whistler number 4, which occurred at a 
radial distance of $R=1.44 R_{N}$ and a magnetic latitude of $\lambda_{m}=21^{\circ}$. This is the best example of a whistler-like signal detected during the entire flyby. Even then the signal is very weak. The maximum electric field strength of the whistler in Figure 2 is estimated to be only 12 $\mu \mathrm{V} / \mathrm{m}$. The narrowband character of the signal, decreasing monotonically in frequency with increasing time, is clearly evident. The frequencytime slope of the signal, $d f / d t$, also decreases with increasing time, as is characteristic of low frequency whistlers in the Earth's magnetosphere (see Helliwell [1965]), and in Jupiter's magnetosphere (see Gurnett et al. [1979]). Figure 3 shows whistlers number 5 and 7 . These signals occur in the same general frequency range as whistler number 4, but over a somewhat narrower frequency range. Whistlers number 6 and 8 also occur on this same spectrogram, but are too weak to be seen in this display.

Eckersley [1935] showed that the arrival time, $t$, of a whistler at a frequency, $f$, is given by a simple relation called the Eckersley law,

$$
t=D / \sqrt{f}+t_{0}
$$

where $D$ is a constant called the dispersion, and $t_{o}$ is the time of the lightning flash. To provide further evidence that the dispersive tones detected by Voyager are in fact whistlers, we have measured the arrival time of each whistler as a function of frequency and fit the Eckersley law to these arrival times. Of the original 16 whistlers, only 8 could be measured with sufficient accuracy to provide reliable least-mean- 
square fits. In general, the fits to the Eckersley law are quite good, although in some cases the frequency range over which measurements could be made is quite limited. The best $f$ it $D$ and $t_{0}$ values are listed in Table 1. To provide an overview of the measured dispersion characteristics, Figure 4 shows the arrival time (dots) for all of the whistlers for which adequate fits could be obtained, plotted on a common time base, $t-t_{0}$. Also shown are some representative dispersion curves (solid lines) computed from the Eckersley law. The results are quite striking. All except whistler number 1 are seen to be bunched together along a narrow range of dispersion curves, with $D$ values ranging from about 24,300 to $29,200 \mathrm{sec} \mathrm{Hz}{ }^{1 / 2}$. Furthermore, even though the frequency range of some of the individual events is too narrow to provide a good test of the $1 / \sqrt{\mathrm{f}}$ law, the overall fit is very convincing. In particular, the slope, $\mathrm{df} / \mathrm{dt}$, of the individual events varies with frequency in almost precisely the manner predicted by the Eckersley law. This good agreement provides convincing evidence that the signals are produced by dispersive whistler-mode propagation from an impulsive source. As far as we know, lightning in the atmosphere of Neptune is the only plausible source of such signals. 


\section{DISPERSION ANALYSIS}

Since the propagation path and dispersion of a whistler depends on the magnetic field topology and plasma density distribution, whistlers can provide useful information on the structure of Neptune's magnetosphere. At low frequencies it can be shown [Storey, 1953] that the ray path of whistler mode waves is confined to within $19^{\circ}$ of the magnetic field direction. Therefore, to a first approximation the ray paths follow a magnetic field line. It can also be shown [Stix, 1962] that the whistler mode cannot propagate at frequencies above the electron cyclotron frequency, $f_{c}=28 \mathrm{~B}(\mathrm{~Hz})$, where $B$ is the magnetic field strength in $\mathrm{nT}$, or above the electron plasma frequency, $f_{p}=$ $9000 \sqrt{\mathrm{N}}(\mathrm{Hz})$, where $\mathrm{N}$ is the electron density in $\mathrm{cm}^{-3}$. Since whistlers were observed at frequencies extending up to about $12 \mathrm{kHz}$, these conditions imply that the absolute minimum magnetic field strength and electron density along the ray path are $B_{\min }=430 n T$ and $N_{\min }=$ $1.8 \mathrm{~cm}^{-3}$. The minimum magnetic field condition indicates that the ray paths must be confined to the near vicinity of the planet. Although the exact magnetic field configuration near the planet is not yet known, we can use the magnetic moment of 0.133 Gauss $R_{N}{ }^{3}$, given by Ness et al. [1989], to estimate that the ray paths cannot extend to radial distances more than about $3 \mathrm{R}_{\mathrm{N}}$. Thus, it is clear that the region where the whistlers were observed is characterized by low L-values 
(most likely $L \leqslant 3$ ). This conclusion is consistent with the radial distances and magnetic latitudes listed in Table 1.

The dispersion of a whistler is controlled by several factors. For the relatively simple Eckersley law to apply, several very restrictive assumptions must be satisfied (see Helliwell [1965]). These include propagation at relatively small angles to the magnetic field, wave frequencies substantially less than the electron cyclotron frequency $\left(f<f_{c}\right.$ ) and electron plasma frequency $\left(f<f_{p}\right)$, and relatively high plasma densities $\left(f_{p}{ }^{2} \gg f f_{c}\right)$. When these conditions are satisfied the dispersion constant $D$ in the Eckersley law is given by the following integral evaluated along the ray path.

$$
D=\frac{1}{2 c} \int \frac{f_{p}}{\sqrt{f_{c}}} d s
$$

Although some uncertainty exists in the ray path and cyclotron frequency because of uncertainties in the magnetic field geometry, by far the largest uncertainty is in the plasma density, which must be known to compute $f_{p}$. Therefore, before attempting to evaluate the dispersion, we must discuss the plasma density.

Relatively little is known about the plasma density in the vicinity of Neptune. For reference a detailed discussion of the plasma density in the magnetosphere and ionosphere of Neptune is given in the Appendix. Basically three types of plasma density measurements are available from Voyager 2, (1) in situ plasma measurements, (2) various 
limits imposed by plasma wave cutoffs and resonances, and (3) radio occultation measurements. In the region near closest approach the in situ plasma measurements from the Voyager plasma instrument [Belcher et al., 1989] give very low plasma densities, typically $10^{-2}$ to $10^{-1} \mathrm{~cm}^{-3}$, with a maximum of $1.4 \mathrm{~cm}^{-3}$. These densities are in direct conflict with the whistler observations, since they give electron plasma frequencies below the whistler frequencies, which is impossible. In fact, since the whistlers show little or no deviation from the Eckersley law, the electron plasma frequency must be well above the upper frequency limit of the whistlers over the entire region where the whistlers are observed, otherwise the signals would have a frequencytime form known as a "nose whistler" [Helliwell, 1965]. A conservative estimate, based on the condition $f_{p}{ }^{2} \gg f f_{c}$ indicates that the electron plasma frequency must be at least 50 to $100 \mathrm{kHz}$, which corresponds to electron density of at least 30 to $100 \mathrm{~cm}^{-3}$. The most likely explanation of the disagreement with the plasma measurements is that the plasma instrument is simply not able to detect the cold, dense plasma that exists near closest approach (see the discussion in the Appendix) .

Unfortunately, the plasma wave cutoffs and resonances observed near closest approach are difficult to analyze and do not provide a clear determination of the electron density. However, they do not preclude electron densities of $100 \mathrm{~cm}^{-3}$, or more. For further details see the discussion in the Appendix. Radio occultation measurements [Tyler et al., 1989], which are characteristic of the ionosphere around 
the limb of the planet, clearly show that electron densities as high $2 \times 10^{3} \mathrm{~cm}^{-3}$ exist in the ionosphere at an altitude of $1400 \mathrm{~km}$, decreasing to several hundred $\mathrm{cm}^{-3}$ at altitudes of $5000 \mathrm{~km}$ ( $R=1.2$ $R_{N}$ ). The radio occultation measurements also show that the lonosphere is very cold. The temperature estimated from the scale height is only $950^{\circ} \pm 160^{\circ} \mathrm{K}$. On the dayside of Neptune the ionospheric electron densities are likely to be even higher. Models of Neptune's ionosphere [Shinagawa and Waite, 1989] give maximum electron densities of $10^{5} \mathrm{~cm}^{-}$ 3 , and comparisons with Uranus indicate that the density could be as high as $6 \times 10^{5} \mathrm{~cm}^{-3}$ (for further details see the discussion in the Appendix) .

Armed with these overall impressions of the electron density in the vicinity of Neptune, we can now return to the evaluation of the whistler dispersion, given by Equation (2). To obtain a rough firstorder estimate we compute the path length, $\ell$, that would be required to obtain a given dispersion, assuming that the electron cyclotron frequency and plasma frequency are constant. The required path length is given by

$$
\ell=2 c \frac{\sqrt{f}}{f_{p}} D
$$

To illustrate the possible extremes, we consider two propagation paths, one through the magnetosphere, where the electron density is relatively low, and the other through the ionosphere, where the electron density 
is relatively high. The assumed parameters are listed in Table 2, along with the corresponding path lengths in Neptune radii $\left(1 R_{N}=\right.$ $24,762 \mathrm{~km})$. For the dispersion we assume $D=26,000 \mathrm{sec} \mathrm{Hz}^{1 / 2}$, which is typical of the whistlers listed in Table 1. For the magnetospheric propagation path we use $f_{c}=28 \mathrm{kHz}(B=1,000 \mathrm{nT})$, which would correspond to a propagation path extending out to about $2.4 R_{N}$ from the center of the dipole. This propagation path gives roughly the lowest cyclotron frequency that would be consistent $\left(f \ll f_{c}\right)$ with the Eckersley law. For the electron density two cases are given. The low density case $\left(\mathrm{N}=30 \mathrm{~cm}^{-3}\right)$ corresponds to roughly the lowest electron plasma frequency that would be consistent $\left(f_{p}{ }^{2} \gg f_{c}\right)$ with the Eckersley law. The high density case represents a relatively high density $\left(10^{3} \mathrm{~cm}^{-3}\right)$ that is comparable to the Io plasma torus at Jupiter. For the ionospheric propagation path we have used the plasma frequency, $7 \mathrm{MHz}$, reported by Zarka and Pedersen [1986] for the dayside ionosphere of Uranus. This value is probably a little high for Neptune (because of the reduced solar UV flux), but not implausible. For the magnetic field strength two cases are given, one ( $B=10,000 \mathrm{nT}$ ) corresponding to the lowest field estimated by Ness et al. [1989] at the planet's surface, due to the offset of the dipole, and the other ( $B=100,000 \mathrm{nT}$ ) corresponding to the highest field at the planet's surface.

As can be seen from Table 2, the required path lengths are very large. They range from $2,159 \mathrm{R}_{\mathrm{N}}$ for the low density magnetospheric path, to $48 R_{N}$ for the weak field ionospheric path. These long path 
lengths either imply much larger plasma densities than are indicated by any of the presently available models or measurements, or a large number of bounces from one hemisphere to the other, or some combination of these effects. From strictly a path length point of view, the ionosphere provides the most favorable propagation path for explaining the large dispersions with the minimum number of bounces. Although the path lengths are very long, they are not ruled out, as far as we know, by any fundamental consideration. Long whistler mode propagation paths, in extreme cases involving as many as 200 bounces from one hemisphere to the other, have been observed in the Earth's magnetosphere (see Figure 4-8 of Helliwell [1965]). The main requirement for a long propagation path is that the plasma temperature must be very low so that Landau damping is negligible. 
IV. PROPAGATION PATH

Since a detailed model of Neptune's magnetic field is not yet available, and very little is known about the electron density distribution around Neptune, no attempts have been made to carry out whistler ray tracing calculations. However, certain general characteristics of the ray paths can be addressed. From the previous dispersion analysis it appears almost certain that many bounces are required from one hemisphere to the other. The reflection at the end of each bounce can be due to either the discontinuity in the index of refraction at the base of the ionosphere, or the refraction of the wave normal angle through $90^{\circ}$ at frequencies below the lower hybrid resonance (LHR) frequency. This latter type of reflection is responsible for what are called "magnetospherically reflected" whistlers [Smith and Angerami, 1968], and could only occur in regions where the surface magnetic field strength exceeds about $18,500 \mathrm{nT}$ (which is possible), so that $\mathrm{f}_{\text {LHR }}>12 \mathrm{kHz}$. In the Earth's magnetosphere multiple bounce (hop) whistlers are frequently observed in which the ray path retraces the same magnetic field line, as in Figure 5. This type of field-aligned propagation is made possible by field-aligned density irregularities that act to duct the signal along the magnetic field line [Helliwell, 1965]. Such ducted field-aligned propagation produces a train of whistlers, each with a progressively larger dispersion, such as illustrated at the bottom of Figure 5. 
Since there is no evidence of a train of whistlers at Neptune it is unlikely that the signals are bouncing from one hemisphere to the other along a single field-aligned duct. Some mechanism must exist for the ray path to shift across the magnetic field lines on successive bounces so that only a single whistler is observed at the spacecraft. Non-ducted whistler propagation is frequently observed in the Earth's magnetosphere [Smith and Angerami, 1968]. For non-ducted propagation the whistler-mode energy can propagate at a substantial angle to the magnetic field direction (up to $19^{\circ}$ ). The resulting ray path can then deviate significantly from the magnetic field lines. If the wave vector is in the meridian plane, the ray path tends to shift to higher and higher L-values on successive bounces, as illustrated in Figure 6. This pattern is characteristic of magnetospherically reflected whistlers. For such ray paths the dispersion should decrease as the spacecraft moves to lower and lower L-values. Since no such systematic trend was observed, it is unlikely that a simple meridional ray path geometry can explain the whistlers observed by Voyager at Neptune. More likely, the ray paths migrate in longitude, as illustrated in Figure 7. This type of longitudinal migration would allow the ray paths to make repeated passes through the dense dayside ionosphere before being detected on the nightside of Neptune, where the density is probably much lower. Even though non-ducted whistlers do not retrace the same ray path on successive bounces, they can still occur in trains [Edgar, 1976]. Non-ducted whistler trains occur because the ray path from the lightning source to the spacecraft has 
multiple solutions. Whether non-ducted propagation can explain the single isolated whistlers observed at Neptune will have to be explored by computer ray tracing calculations.

Finally, we should point out that the magnetic field geometry may also play an important role in producing the large dispersion. Since the largest electron densities probably occur in the ionosphere, any magnetic field configuration that tends to maintain nearly horizontal magnetic field lines through the ionosphere will result in ray paths with large dispersions. The offset of the magnetic dipole from the center of the planet may help produce this type of configuration. These and other ray path considerations will have to be explored with detailed ray tracing calculations when a suitable magnetic field model becomes available. 
- 


\section{CONCLUSIONS}

In this paper we have presented evidence for the existence of whistlers in Neptune's magnetosphere. The observed signals consist of a narrowband tone that decreases monotonically with increasing time. Sixteen such events were observed, all near closest approach at low magnetic latitudes. The frequency-time characteristics of these signals fit the Eckersley law, which provides strong evidence that the signals are produced by lightning in the atmosphere of Neptune. The upper frequency limit of the whistlers, $12 \mathrm{kHz}$, is in direct conflict with the Voyager 2 plasma measurements, which gives electron densities too low for the whistler mode to propagate. Most likely the plasma instrument is simply not able to detect the cold, dense plasma that would be required for whistler mode propagation in this region. The dispersion of the whistlers is also very large $\left(D=26,000 \operatorname{sec~Hz}^{1 / 2}\right)$, which indicates substantial plasma densities somewhere along the propagation path, and/or a large number of bounces from one hemisphere to the other. The most likely propagation path probably involves a lightning source on the dayside of the planet, with repeated bounces through the dense dayside ionosphere at low L-values to accumulate the required dispersion. Detailed computer ray tracing calculations using suitable magnetic field and plasma density models will be required to investigate the ray paths involved. The signals are also very weak, much weaker than the whistlers observed in Jupiter's magnetosphere 
(after taking into account the radial distances involved). The low intensities, or possibly other geometric considerations may explain why no lightning was detected by either the imaging [Smith et al., 1989] or radio astronomy [Warwick et al., 1989] instruments. 


\section{APPENDIX}

Since the plasma density plays a crucial role in the propagation of whistlers, it is useful to briefly summarize what is known about the plasma density in Neptune's magnetosphere and ionosphere.

Plasma Measurements. The only direct in situ plasma density measurements are from the Voyager 2 plasma instrument [Belcher et al., 1989]. In general the plasma densities reported from the plasma instrument are very low, typically $10^{-3}$ to $10^{-1} \mathrm{~cm}^{-3}$. The maximum plasma density was either 0.6 or $1.4 \mathrm{~cm}^{-3}$, depending on whether $\mathrm{H}^{+}$or $\mathrm{N}^{+}$is assumed to be the dominant ion. These maximum values were obtained at 0420 SCET, in the same region where the whistlers were observed. All of the plasma density measurements obtained in the region where whistlers were observed are in direct conflict with the whistler observations. The reason is that the electron plasma frequency computed from the plasma density measurements is below the maximum frequency of the whistlers, which cannot occur for the whistler mode of propagation. The most likely explanation of this discrepancy is that the plasma instrument was not measuring the total plasma in the region near closest approach. During the pass over the polar region, the plasma sensor was oriented in such a way as to optimize the detection of precipitated auroral particles. In this orientation, the sensor is relatively insensitive to plasma arriving from the ram 
direction, particularly if the plasma is very cold [personal communication, J. Belcher, 1990].

Plasma Densities From Plasma Wave Resonances and Cutoffs. It is well known that various cutoffs and resonances in the plasma wave spectrum can be used to determine the plasma density. In fact, electrostatic emissions at the upper hybrid resonance (UHR) have been found to be in good agreement with the in situ plasma measurements near the inbound and outbound equator crossings at $10 \mathrm{R}_{\mathrm{N}}$ and $11 \mathrm{R}_{\mathrm{N}}$, respectively [Gurnett et al., 1983; Barbosa et al., 1990]. Unfortunately, in the region around closest approach the plasma wave spectrum provides very little definitive information on the plasma density. A spectrogram of the plasma wave electric field intensities in this region is shown in the bottom panel of Figure 8 . This spectrogram is essentially an enlarged version of the high frequency part of Figure 5 from Gurnett et al. [1989]. No obvious resonances, such as electron plasma oscillations or upper hybrid emissions, are present that can provide a direct indication of the electron density. At frequencies from 10 to $50 \mathrm{kHz}$ the spectrum is dominated by radio emissions. For a discussion of these radio emissions, see Kurth et al. [1990]. On the inbound pass the radio emission at about $50 \mathrm{kHz}$ has a sharply defined cutoff at about 0325 SCET, slightly above the electron cyclotron frequency. This cutoff has been identified by Kurth et al. as the $f_{R=0}$ cutoff associated with the free space $R-X$ mode. The fact that the cutoff is very close to the electron cyclotron frequency indicates that the electron plasma frequency is well below $50 \mathrm{kHz}$ at 
this point. A second cutoff can be seen below the electron cyclotron frequency from 0300 to 0345 SCET at about $10 \mathrm{kHz}$. At 0345 SCET the cutoff increases rapidly, reaching $56 \mathrm{kHz}$ at about 0352 SCET. This cutoff could be caused by the low frequency cutoff of the free space L-O mode, or the $f_{L=0}$ cutoff of the 2 -mode. In either case the cutoff only gives an upper limit to the electron plasma frequency, since there is no guarantee that waves actually reach the cutoff. Of these, the 2 -mode gives the upper limit to the plasma frequency. This limit is given by $f_{p}(\operatorname{Max})=\left[f_{L=0}\left(f_{L=0}+f_{c}\right)\right]^{1 / 2}$, where $f_{L=0}$ is the low frequency cutoff of the noise band. The solid line labelled $f_{p}$ (Max) shows this upper limit. The electron plasma frequency must lie in the shaded region below $f_{p}(\operatorname{Max})$.

In the crucial time period from 0405 to 0425 SCET, where most of the whistlers were observed, the high frequency radio emissions are almost entirely absent. This could indicate either that the electron plasma frequency is quite high, much greater than $50 \mathrm{kHz}$, which would block the radio emission via the $f_{L=0}$ cutoff, or that the spacecraft has dropped below the propagation horizon of a remote radio source. Although no radio emissions are present in this region, a weak plasma wave emission can be seen extending from $1 \mathrm{kHz}$ to as high as $50 \mathrm{kHz}$. Since this noise occurs in the same frequency range as the whistlers, it must be propagating in the whistler mode. Most likely it is similar to plasmaspheric hiss in the Earth's magnetosphere [Thorne et al., 1973]. Since the whistler mode cannot propagate at frequencies above the electron plasma frequency, this noise places a lower limit on the 
plasma frequency. This lower limit, labelled $f_{p}$ (Min), is shown in Figure 5. As can be seen, this limit indicates that the electron plasma frequency probably extended well above $50 \mathrm{kHz}$ in the region near closest approach. At the magnetic equator crossing (0420 SCET) Sawyer et al. [1990] have reported a narrow, well-defined burst of waves at frequencies from 104 to $212 \mathrm{kHz}$ that they identify as electrostatic $(n+1 / 2) f_{c}$ waves. If these waves are associated with the upper hybrid resonance, as is often the case, then the electron plasma frequency is probably $200 \mathrm{kHz}$, or more, in this region.

On the outbound pass the high frequency radio emission again reappears above the electron cyclotron frequency after about 0500 SCET. A continuation of this radio emission appears to extend below the electron cyclotron frequency from about 0423 to 0515 SCET. Although this noise has intensities comparable to the high frequency radio emission, which suggests that it is free space L-O mode radiation, we believe that this noise is $Z$-mode radiation [Kurth et al., 1990]. The reason is that in the wideband frame at 0424:47 SCET a whistler can be seen extending above the low frequency cutoff of this noise. This situation cannot occur for the free space $L-0$ mode, since the electron plasma frequency, which is the low frequency cutoff of the L-O mode, would have to be below the frequency of the whistler. Therefore, the noise must be $\mathrm{Z}$-mode radiation. This interpretation is further supported by the fact that the noise has an upper cutoff that follows the electron cyclotron frequency (see Figure 8 ), very similar to 2 -mode radiation in the Earth's magnetosphere [Gurnett et al., 1983]. Based 
on this interpretation, the low frequency cutoff of the noise band can then be used to place an upper limit on the electron plasma frequency, following the same procedure as on the inbound pass. This limit is shown by the solid line labelled $f_{p}(\operatorname{Max})$, on the right-hand side of Figure 8. From the limits given in the top panel, we conclude that the electron plasma frequency started from somewhat less than $10 \mathrm{kHz}$ on the inbound pass, increased to well over $50 \mathrm{kHz}$ (possibly as high as 200 $\mathrm{kHz}$, or more) near closest approach, and then dropped to less than 10 $\mathrm{kHz}$ on the outbound pass.

Radio Occultation Measurements. The third method of measuring the plasma density in Neptune's magnetosphere is provided by the Voyager radio occultation experiment [Tyler et al., 1989]. These measurements were obtained as the radio signal from the spacecraft to Earth was occulted by the limb of the planet. By analyzing the phase shift of the received signal, a profile of the ionospheric electron density can be obtained as a function of altitude. This profile has a maximum electron density of $\mathrm{N}_{\max }=2 \times 10^{3} \mathrm{~cm}^{-3}$ at an altitude of $1400 \mathrm{~km}$, and a scale height of about $1800 \mathrm{~km}$. The scale height indicates that the plasma is extremely cold. If $\mathrm{H}+$ is the dominant ion the temperature is $950^{\circ} \pm 160^{\circ} \mathrm{K}$. Electron densities on the order of several hundred $\mathrm{cm}^{-3}$ were detectable at altitudes up to $5000 \mathrm{~km}\left(\mathrm{R}=1.2 \mathrm{R}_{\mathrm{N}}\right)$. These electron densities are considerably larger than those detected by the plasma instrument at a comparable radial distance.

Since the ray path of the radio occultation signal passed through the ionosphere near the terminator, where the solar illumination angle 
is very low, these densities are not necessarily characteristic of the highest electron densities that could exist in the ionosphere of Neptune. The highest densities would be expected on the dayside of Neptune, near the subsolar point, where the incident solar illumination is the strongest. The best estimate of the dayside ionospheric electron densities is from Uranus, where the low frequency cutoff of radio signals from lightning [Zarka and Pedersen, 1986] can be used to measure the ionospheric electron density. The dayside electron densities inferred at Uranus using this technique give $N_{\max }=6 \times 10^{5}$ $\mathrm{cm}^{-3}$, which corresponds to an electron plasma frequency of $\mathrm{f}_{\mathrm{p}}=7 \mathrm{MHz}$. Since the atmospheres of Neptune and Uranus are expected to be quite similar, and the difference in the solar ultraviolet flux is not large, one would expect the dayside electron density to be somewhat comparable at Neptune. In fact, recent models of the ionosphere of Neptune [Shinagawa and Waite, 1989] give a maximum electron density of $\mathrm{N}_{\max }=$ $10^{5} \mathrm{~cm}^{-3}$, which corresponds to an electron plasma frequency of $f_{p}=2.8$ $\mathrm{MHz}$. 
ACKNOWLEDGEMENTS

The authors would like to thank J. Belcher, M. Kaiser, R. McNutt, and $N$. Ness for several useful discussions concerning the interpretation of these data.

This research was supported by NASA through contract 957723 with the Jet Propulsion Laboratory. 



\section{REFERENCES}

Barbosa, D. D., W. S. Kurth, I.H. Cairns, and D. A. Gurnett, Electrostatic ion and electron cyclotron harmonic waves in Neptune's magnetosphere, Geophys. Res. Lett., in press, 1990.

Barkhausen, H., Zwei mit Hilfe der neuen Verstarker entdeckte Erscheinungen, Physik Z., 20, 401, 1919.

Belcher, J.W., et al., Plasma observations near Neptune: Initial results from Voyager 2, Science, 246, 1478, 1989.

Borucki, W. J., Predictions of lightning activity at Neptune, Geophys. Res. Lett., 16, 937, 1989.

Eckersley, T. L., Musical atmospherics, Nature, 135, 104, 1935.

Edgar, B. C., The upper- and lower-frequency cutoffs of magnetospherically reflected whistlers, J. Geophys. Res., 81, 205, 1976.

Gurnett, D. A., et al., First plasma wave observations at Neptune, Science, 246, 1494, 1989.

Gurnett, D. A., R. R. Shaw, R. R. Anderson, and W. S. Kurth, Whistlers observed by Voyager 1: Detection of lightning on Jupiter, Geophys. Res. Lett., 6, 511, 1979.

Gurnett, D. A., S. D. Shawhan, and R. R. Shaw, Auroral hiss, Z-mode radiation and auroral kilometric radiation in the polar magnetosphere: DE-1 observations, J. Geophys. Res., 88,329 , 1983. 
Helliwell, R. A., Whistlers and Related Ionospheric Phenomena, Stanford Univ. Press, Stanford, 1, 1965.

Kaiser, M. L., J. E. P. Connerney, and M. D. Desch, Atmospheric storm explanation of Saturnian electrostatic discharges, Nature, 303 , $50,1983$.

Kurth, W. S., B. D. Strayer, D. A. Gurnett, and F. L. Scarf, A summary of whistlers observed by Voyager 1 at Jupiter, Icarus, 61, 497, 1985.

Kurth, W. S., D. D. Barbosa, D. A. Gurnett, R. L. Poynter, and I. H. Cairns, Low-frequency radio emissions at Neptune, Geophys. Res. Lett.,. in press, 1990.

Menietti, J. D., and D. A. Gurnett, Whistler propagation in the Jovian magnetosphere, Geophys. Res. Lett., I, 49, 1980.

Ness, N. F., M. H. Acuna, L. F. Burlaga, J. E. P. Connerney, R. P. Lepping, and F. N. Neubauer, Magnetic fields at Neptune, Science, 246, 1473,1989 .

Sawyer, C., J. W. Warwick, and J. H. Romig, Smooth radio emission and a new emission at Neptune, Geophys. Res. Lett., submitted, 1990.

Scarf, F. L., and D. A. Gurnett, A plasma wave investigation for the Voyager mission, Space Sci. Rev., 21, 289, 1977.

Scarf, F. L., D. A. Gurnett, and W. S. Kurth, Jupiter plasma wave observations: An initial Voyager 1 overview, Science, 204, 991, 1979.

Shinagawa, H., and J. H. Waite., Jr., The ionosphere of Neptune, Geophys. Res. Lett., 16, 945, 1989. 
Smith, R. L., and J. J. Angerami, Magnetospheric properties deduced from OGO 1 observations of ducted and nonducted whistlers, J. Geophy. Res., 73, 1, 1968.

Smith, B. A., et al., The Jupiter system through the eyes of Voyager 1 , Science, 204, 951, 1979.

Stix, T. H., The Theory of Plasma Waves, McGraw-Hill, 40, 1962.

Storey, L. R. O., An investigation of whistling atmospherics, Phil. Trans. Roy. Soc. (London), A, 246, 113, 1953.

Thorne, R. M., E. J. Smith, R. K. Burton, and R. E. Holzer, Plasmaspheric hiss, J. Geophys. Res., 78, 1581, 1973.

Tyler, G. L., et al., Voyager radio science observations of Neptune and Triton, Science, 2466, 1466, 1989 .

Warwick, J. W., et al., Voyager planetary radio astronomy at Neptune, Science, $246,1498,1989$.

Zarka, P., and B. M. Pedersen, Radio detection of Uranian lightning by Voyager 2, Nature, 323, 605, 1986. 

TABLE 1

NEPTUNE WHISTLERS

\begin{tabular}{|c|c|c|c|c|c|c|c|}
\hline No. & $\underline{\text { Time }}$ & ${\underline{\mathrm{R}}\left(\mathrm{R}_{\mathrm{N}}\right)}$ & Lat. & $\begin{array}{l}\text { Mag. } \\
\text { Lat. }\end{array}$ & $\underline{D\left(\mathrm{sHz}^{1 / 2}\right)}$ & $\underline{t}_{0}$ & $\begin{array}{c}\text { Frequency } \\
\text { Range } \\
(\mathrm{kHz}) \\
\end{array}$ \\
\hline 1 & $0405: 35$ & 1.30 & $61^{\circ}$ & $33^{\circ}$ & 45,462 & $0356: 59$ & $7.4-7.8$ \\
\hline 2 & $0406: 05$ & 1.31 & $59^{\circ}$ & $32^{\circ}$ & -- & -- & $9.3-10.2$ \\
\hline 3 & $0408: 22$ & 1.37 & $54^{\circ}$ & $27^{\circ}$ & -- & -- & $11.0-11.5$ \\
\hline 4 & $0410: 35$ & 1.44 & $49^{\circ}$ & $21^{\circ}$ & 24,748 & $0406: 43$ & $8.9-11.6$ \\
\hline 5 & $0412: 50$ & 1.51 & $44^{\circ}$ & $16^{\circ}$ & 26,361 & $0408: 30$ & $9.7-10.4$ \\
\hline 6 & $0412: 57$ & 1.51 & $44^{\circ}$ & $16^{\circ}$ & -- & -- & $10.6-11.2$ \\
\hline 7 & $0413: 07$ & 1.51 & $44^{\circ}$ & $16^{\circ}$ & 29,207 & $0408: 40$ & $10.8-11.9$ \\
\hline 8 & $0413: 27$ & 1.52 & $43^{\circ}$ & $14^{\circ}$ & - & -- & $11.3-12.0$ \\
\hline 9 & $0415: 49$ & 1.62 & $39^{\circ}$ & $10^{\circ}$ & -- & -- & $11.4-12.0$ \\
\hline 10 & $0415: 54$ & 1.62 & $39^{\circ}$ & $10^{\circ}$ & -- & -- & $10.8-12.0$ \\
\hline 11 & $0417: 42$ & 1.68 & $36^{\circ}$ & $6^{\circ}$ & 26,103 & $0413: 43$ & $10.3-12.0$ \\
\hline 12 & $0422: 26$ & 1.87 & $30^{\circ}$ & $-2^{\circ}$ & 27,531 & $0417: 20$ & $7.4-8.2$ \\
\hline 13 & $0422: 47$ & 1.88 & $29^{\circ}$ & $-3^{\circ}$ & 29,209 & $0418: 22$ & $10.0-11.8$ \\
\hline 14 & $0422: 51$ & 1.88 & $29^{\circ}$ & $-3^{\circ}$ & 24,330 & $0417: 58$ & $6.1-6.9$ \\
\hline 15 & $0424: 51$ & 1.97 & $27^{\circ}$ & $-6^{\circ}$ & -- & -- & $6.3-6.8$ \\
\hline 16 & $0425: 30$ & 1.99 & $26^{\circ}$ & $-7^{\circ}$ & -- & -- & $11.1-12.0$ \\
\hline
\end{tabular}


TABLE 2

REQUIRED PATH LENGTHS*

\begin{tabular}{c|c|c|c|c|c} 
Path & $\mathrm{N}$ & $\mathrm{B}$ & $f_{\mathrm{p}}$ & $f_{\mathrm{c}}$ & $\ell$ \\
\hline- & $\mathrm{cm}^{-3}$ & $\mathrm{nT}$ & $\mathrm{kHz}$ & $\mathrm{kHz}$ & $\mathrm{R}_{\mathrm{N}}$ \\
Magnetospheric & & & & & \\
(Low Density) & 30 & $10^{3}$ & 49 & 28 & 2,159 \\
(High Density) & $10^{3}$ & $10^{3}$ & 270 & 28 & 392 \\
Ionospheric & - & - & - & - & - \\
(Weak Field) & $6 \times 10^{5}$ & $10^{4}$ & 7,000 & 280 & 48 \\
(Strong Field) & $6 \times 10^{5}$ & $10^{5}$ & 7,000 & 2,800 & 151
\end{tabular}

*Using $\mathrm{D}=26,000 \mathrm{sec} \mathrm{Hz}$ 


\section{FIGURE CAPTIONS}

Figure 1 The trajectory of Voyager II in magnetic latitude, $\lambda_{m}$, and radial distance, $R$, coordinates. The magnetic latitude was computed using the OTD 2 magnetic field model [Ness et al., 1989]. The circles indicate wideband frames, with and without whistlers. The whistlers are numbered from 1 to 16 .

Figure 2 A frequency-time spectrogram showing whistler No. 4. This is the the strongest and clearest whistler detected during the flyby. Even in this case the signal is very weak, only slightly above the receiver noise level.

Figure 3 A frequency-time spectrogram showing whistlers No. 5 and 7.

Figure 4 A plot of frequency, $f$, versus time delay, $t-t_{0}$, for all of the whistlers for which it was possible to measure the time delay. The reference time $t_{0}$ was calculated independently for each whistler using the Eckersley law.

Figure 5 For ducted field-aligned propagation a lightning flash produces a train of whistlers, each with progressively larger dispersion. This type of propagation does not account for the Neptune whistler observations, since all except one of the whistlers have essentially the same dispersion. 
Figure 6 A representative ray path for non-ducted propagation in a meridian plane. This type of propagation also does not appear to account for the Voyager observations, since the dispersion would be expected to decrease with decreasing L-value.

Figure $7 \quad$ Non-ducted propagation can also allow the ray path to migrate in longitude. This type of ray path would allow whistlers from a lightning source on the dayside to reach the nightside of Neptune, which is where Voyager was located when the whistlers were observed. A dayside ray path geometry is favored because of the much higher electron density (hence dispersion) in the dayside ionosphere.

Figure 8 An analysis of the maximum and minimum electron plasma frequency, $f_{p}$, from observations of radio emissions and whistler-mode noise observed near closest approach. The electron plasma frequency must lie in the shaded region of the top panel. The location of the $(n+1 / 2) f_{c}$ emissions is from Sawyer et al. [1990]. 


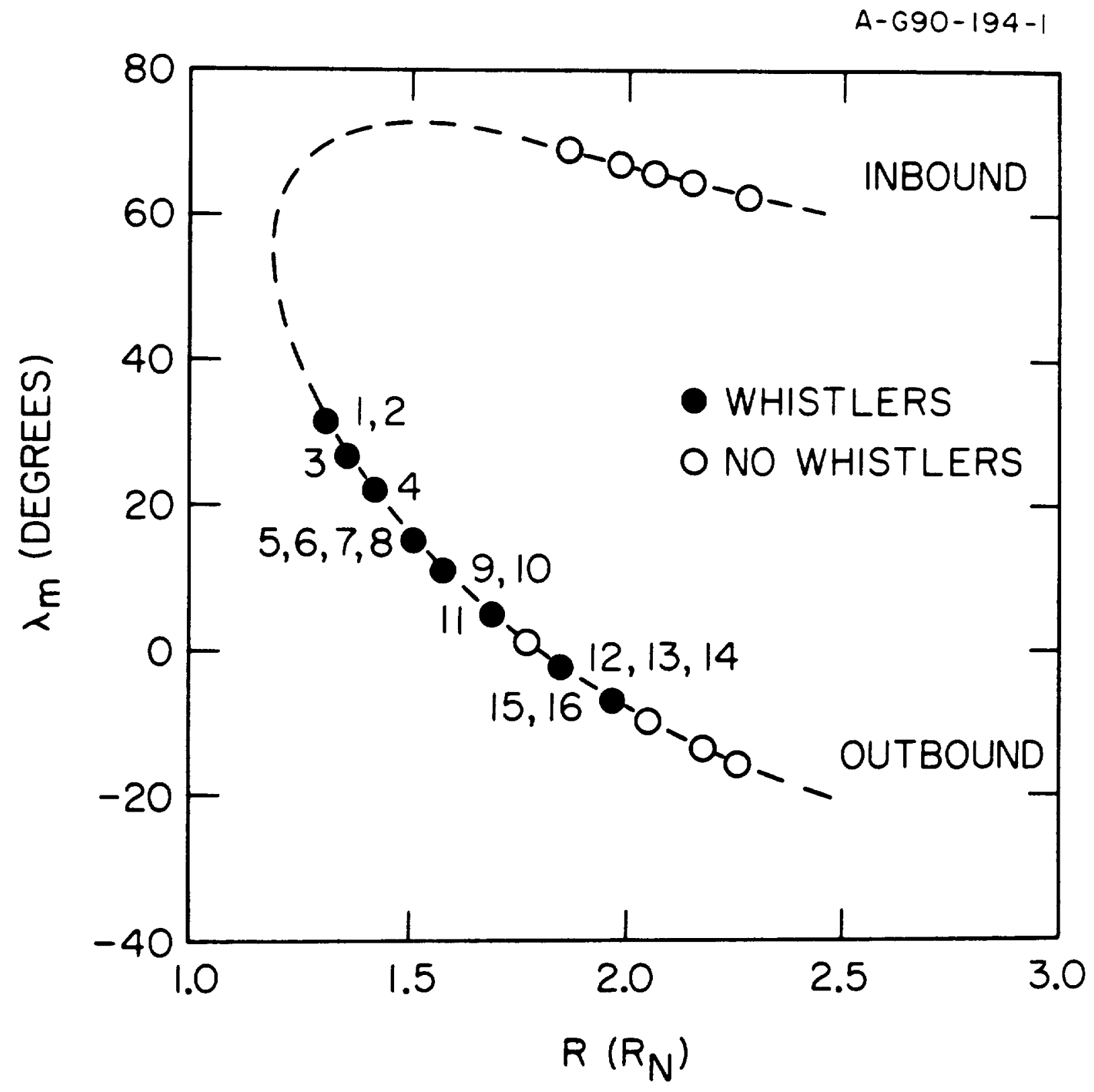

Figure 1 



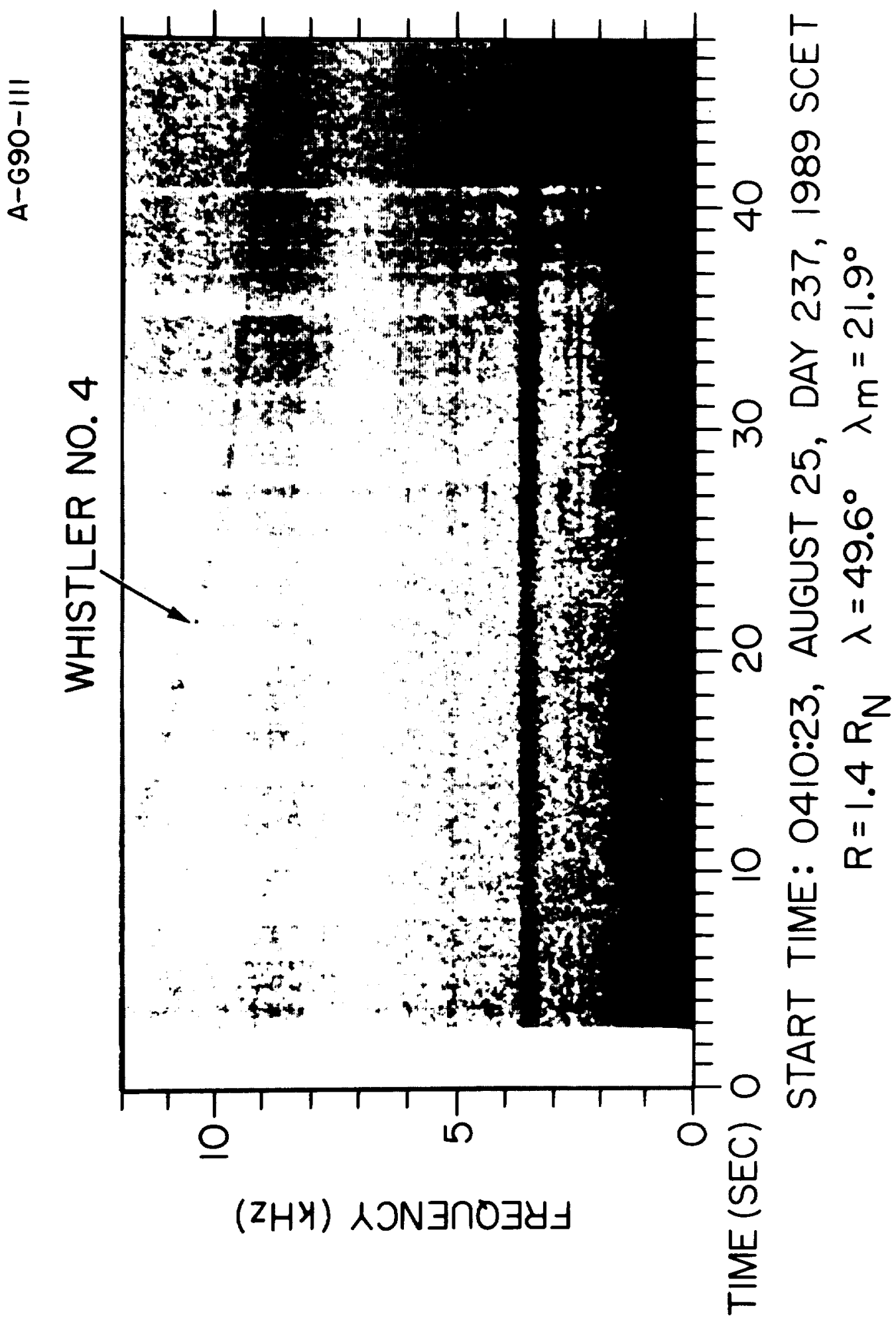

$\underset{\sim}{N}$ 



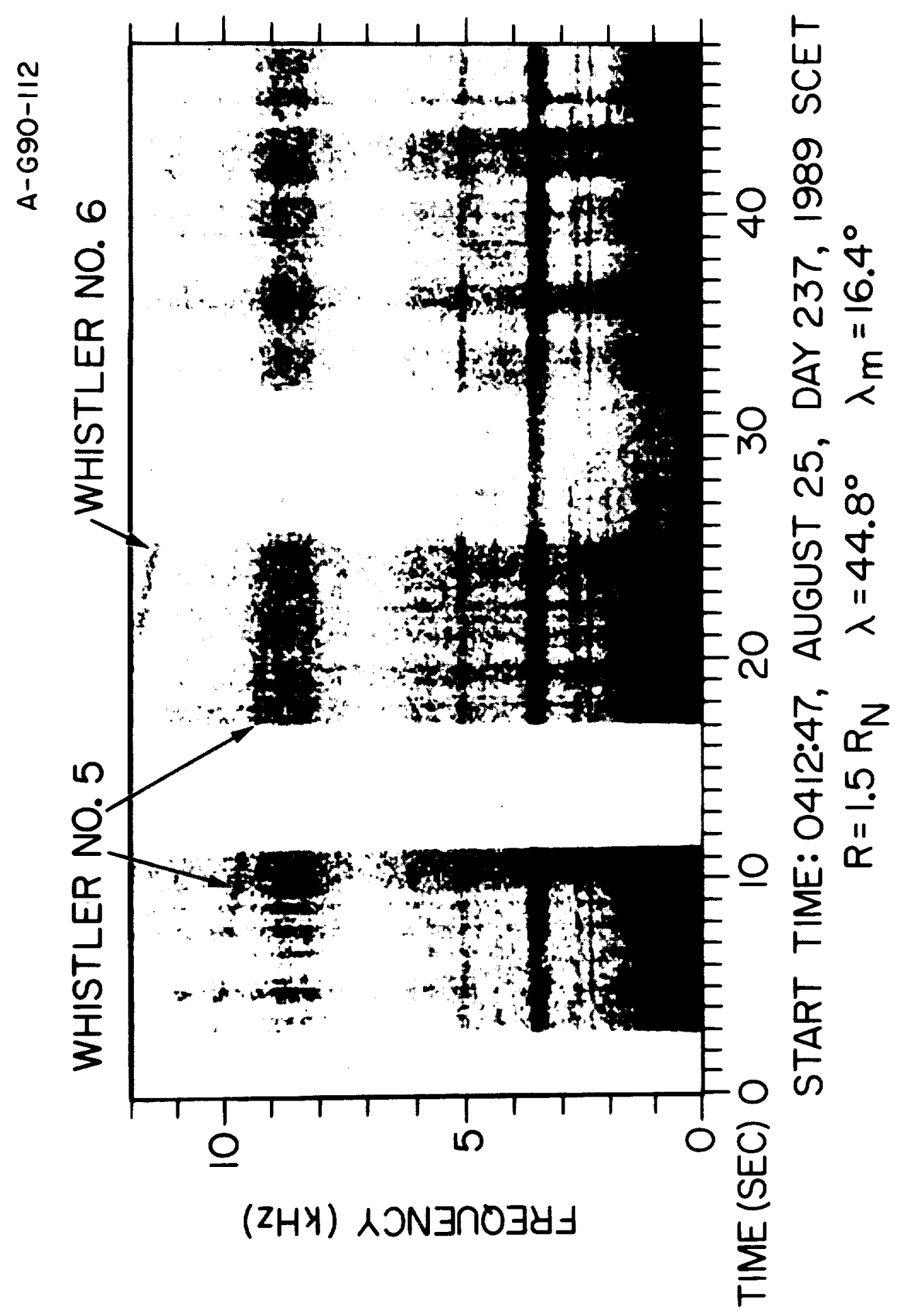

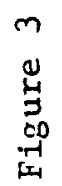





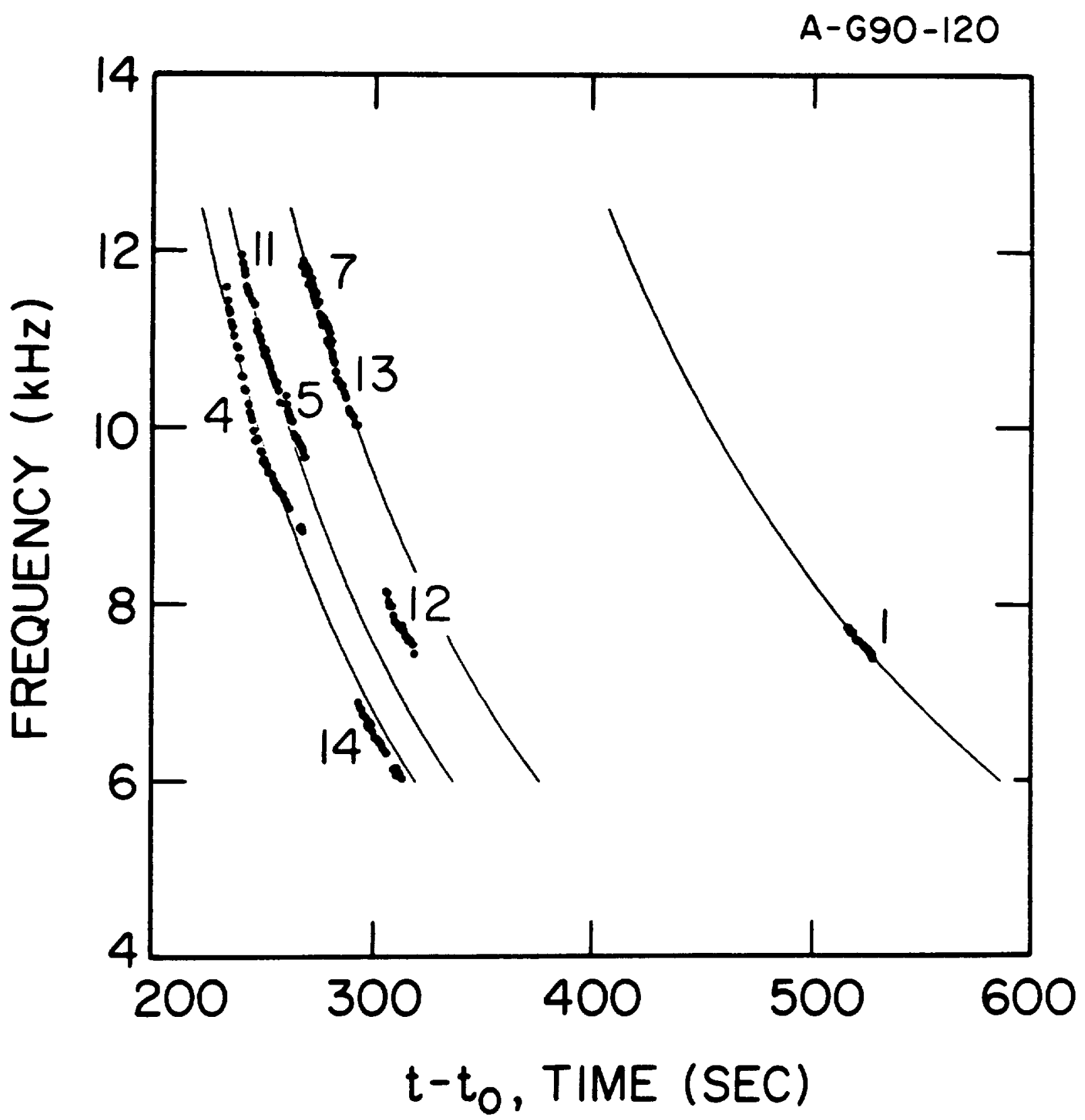

Figure 4 



\section{$A-690-211$}

FIELD-ALIGNED PROPAGATION

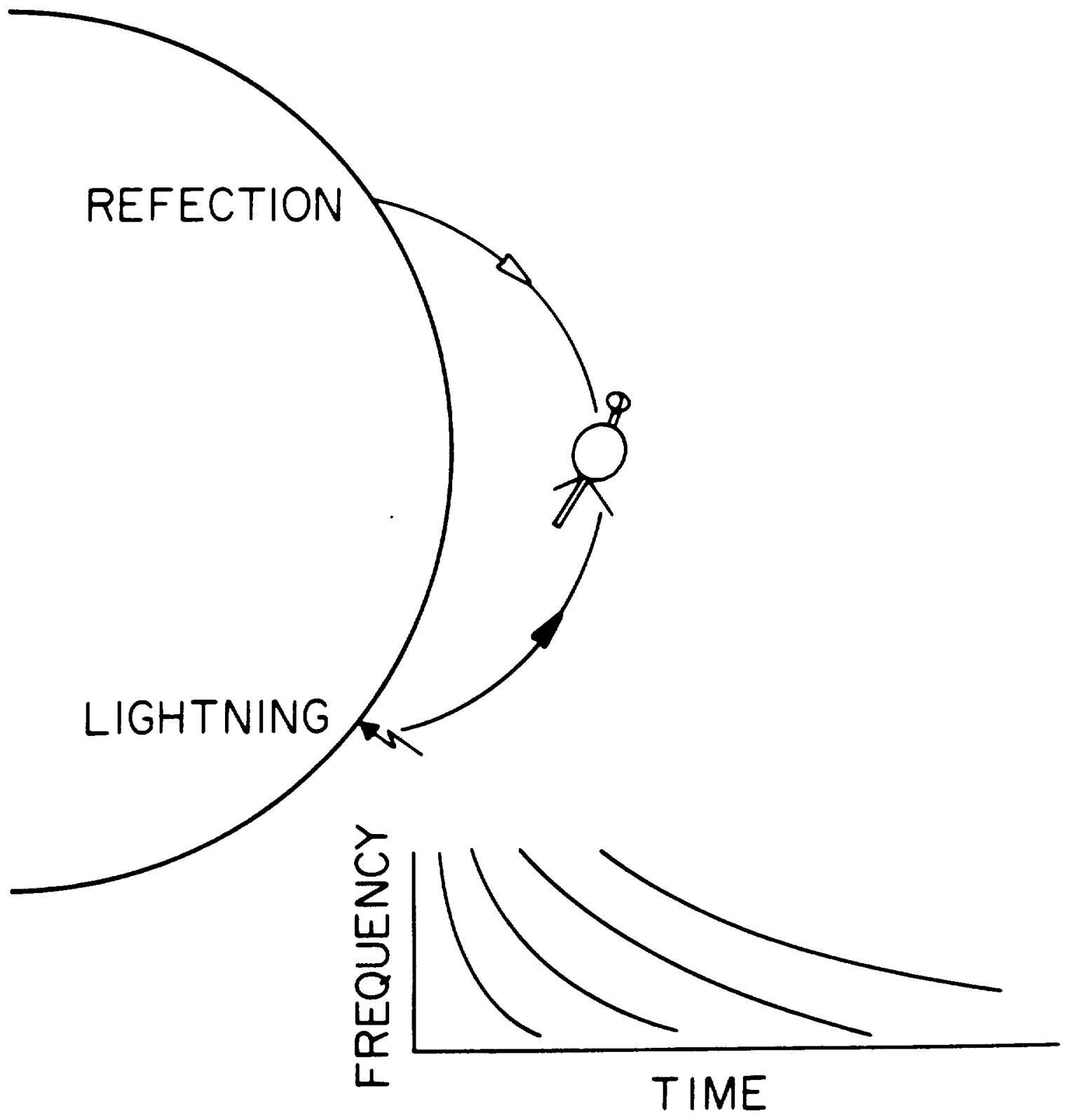

Figure 5 

$A-G 90-212$

MERIDIONAL PROPAGATION

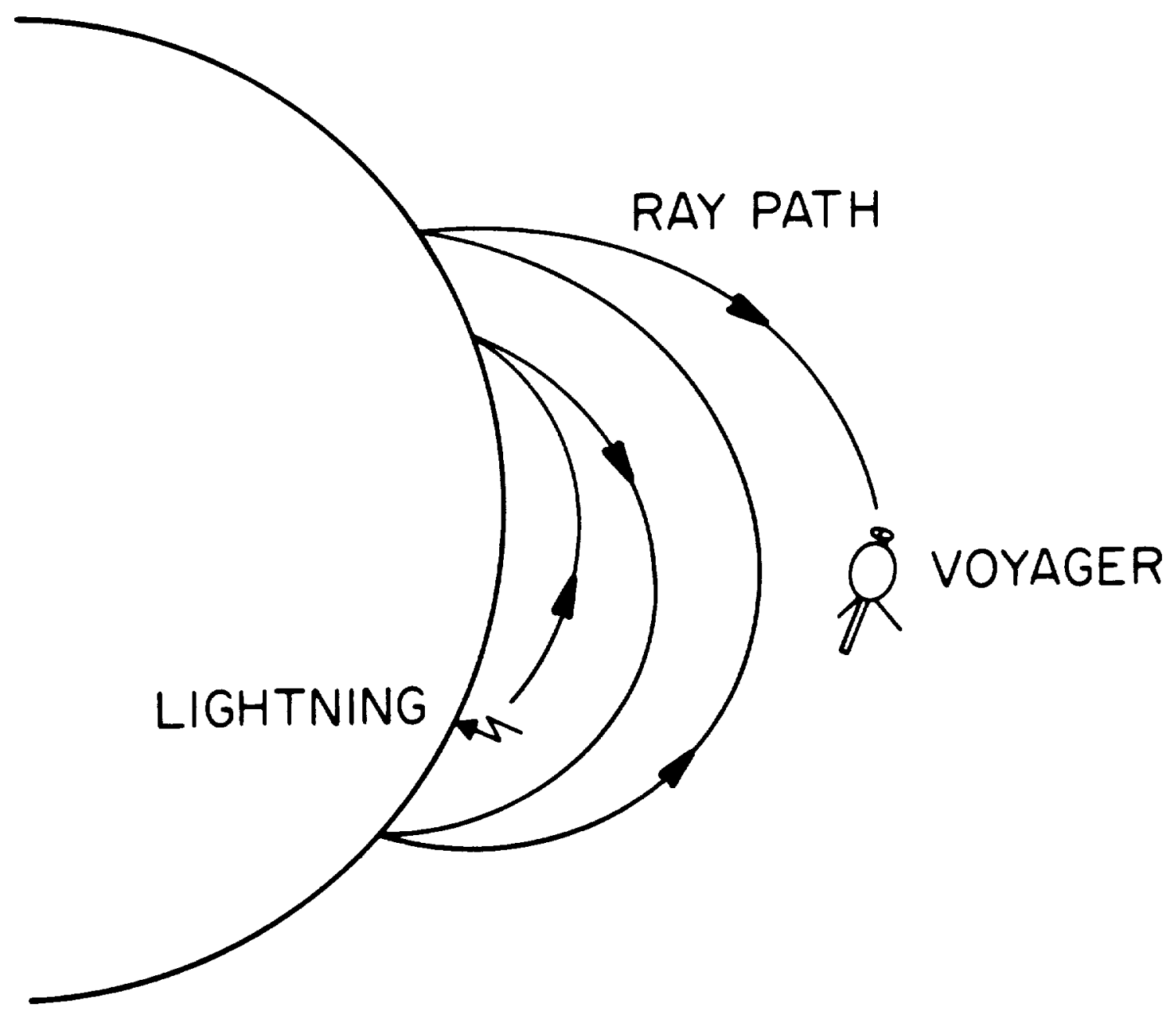





$$
A-690-213
$$

\section{LONGITUDINAL PROPAGATION}

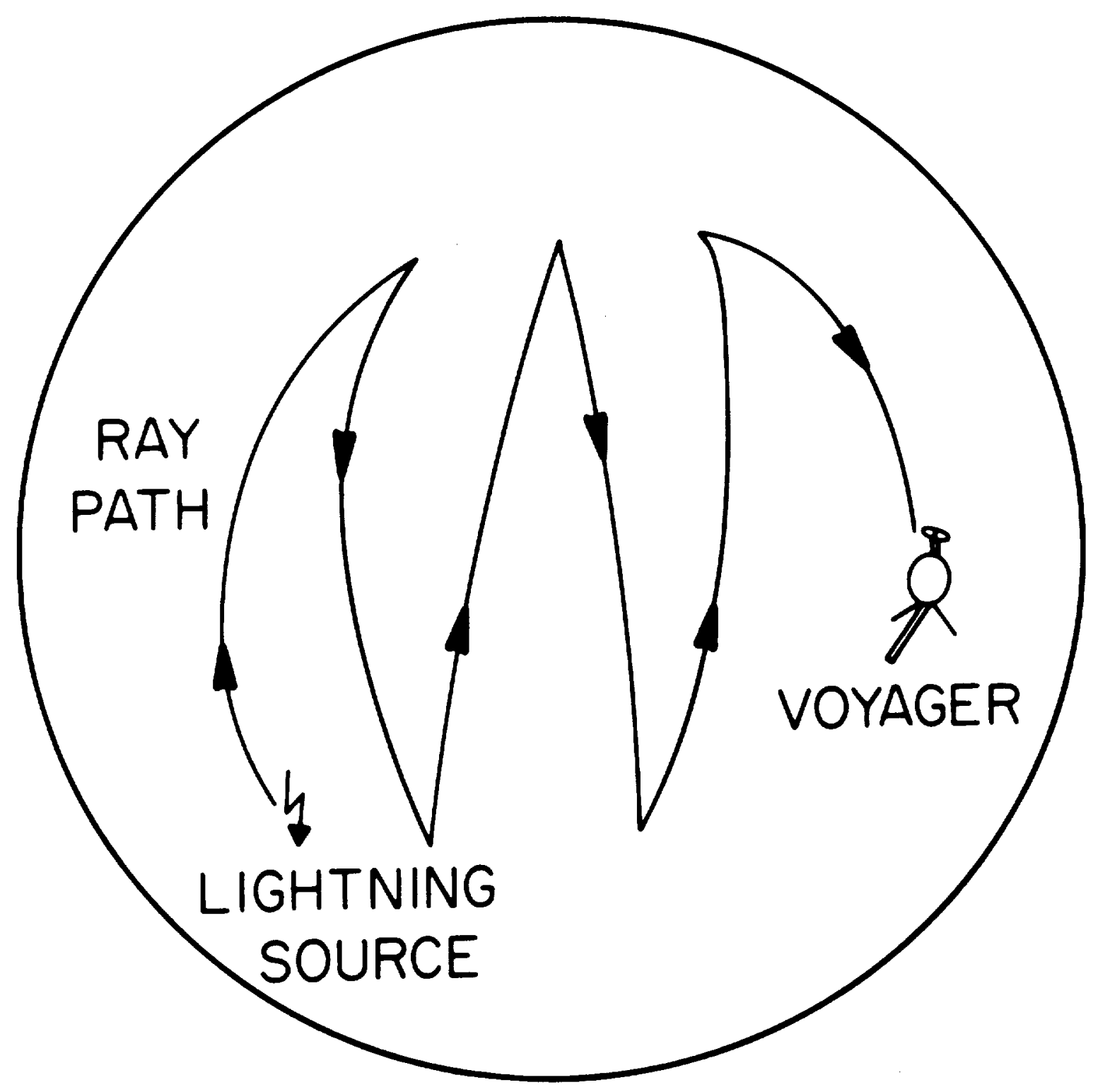

Figure 7 



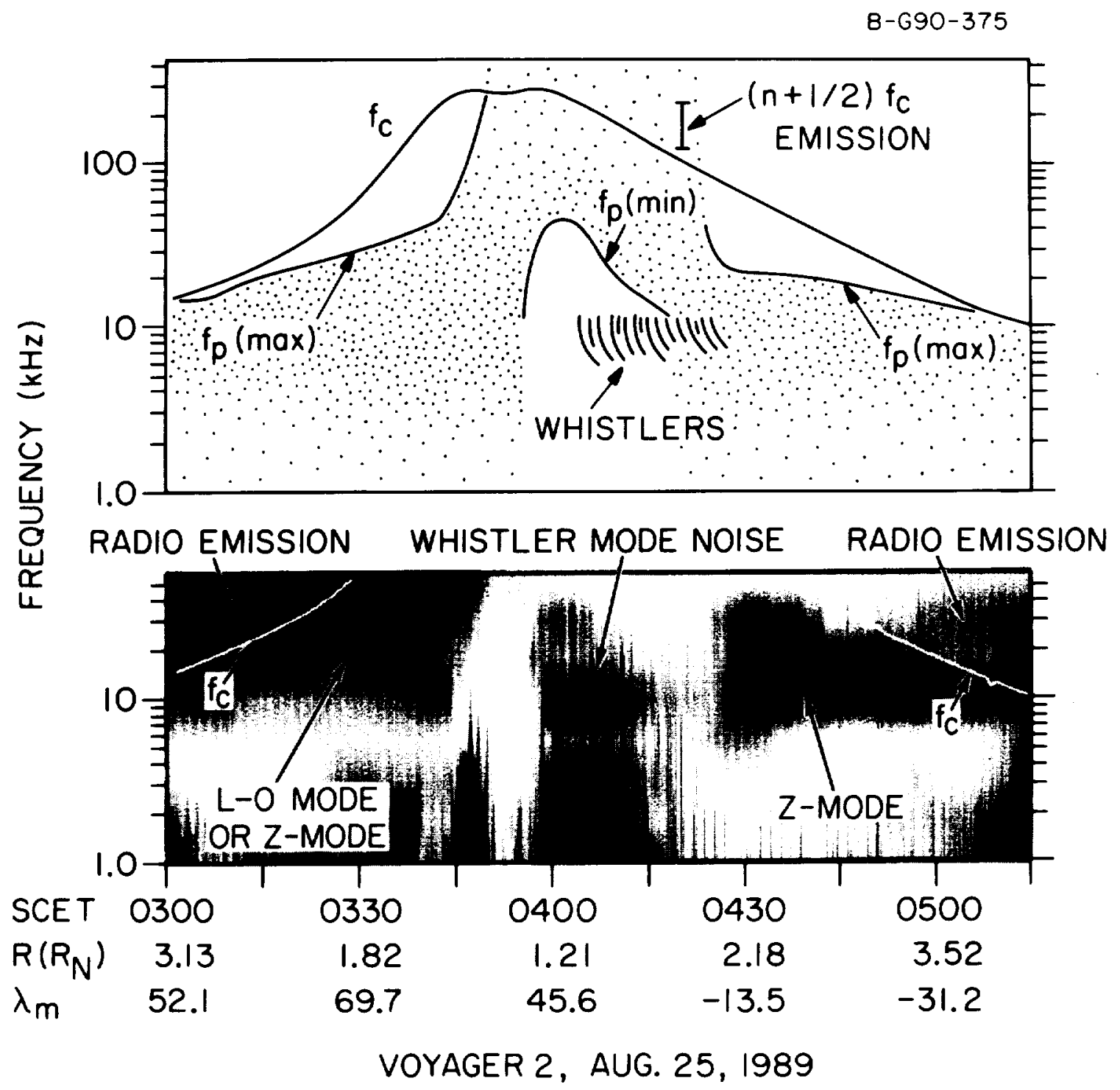

ORlGHA:

OF POOF Chin 


